\title{
A study on theory and physical mechanism of an infinitesimal current-carrying point charge
}

\author{
Nannan Zhou, Guoqiang Xue, Kang Chen \\ Key laboratory of mineral resources \\ Institute of Geology and Geophysics, CAS \\ Beijing, China
}

\begin{abstract}
In antenna theory, the dipole hypothesis is applicable and widely used for observing the far-field radiation. However, when applied to near-field quasi-static radiation observation in geophysical surveying, the transceiver distance is often small, and thereby the source should not be treated as a dipole to avoid misfit between the observation and the modeling based on the dipole theory. To overcome such limitation, this paper first presents a new method of estimating the field generated from a dipole by dividing it into a series of infinitesimal point charge and computing both the velocity field excited by uniform-motion charge and accelerated-velocity field from accelerated charge, so that not only the far-field radiation but also the near-field quasi-static radiation can be accurately estimated. The results are well verified by the traditional methods of radiation modeling from a dipole. Then this study investigates the associated physical mechanism by developing a new equation for computing the radiated field in time-domain based on the frequency-to-time transformation, from which the relationship between a motive charge and its corresponding field is investigated. It is concluded that the field generated from dipole is in nature the same as that generated from motive charge.
\end{abstract}

Keywords-Electric dipole; Radiation field; Point charge; Near-source field

\section{INTRODUCTION}

In antenna theory, far-field radiation generated from a dipole is very useful for remote communication and a dipole is often used to approximate the antenna, because the antenna length is far smaller than the distance between source and receivers. Therefore, at the early stage of communication, the finite-length antenna is treated as a dipole that consists of two equivalent charges with opposite polarity. When the antenna is electrified with alternating currents, the charges start moving with a certain acceleration and velocity; thereby a series of elementary current are generated.

When applied to geophysical exploration, if length of the grounding-wire source and circle-loop source is small, they can be regarded as electric dipole source and magnetic dipole source, respectively. The function of source potential is derived by substituting the magnetic moment of dipole source into the Green Function, without taking into account the effect of varying transceiver distance due to the infinite-length antenna instead of a point charge. For long grounding-wire source and large loop source, the generated potential functions are often computed as an integration of a series of diploe sources, rather than point sources ${ }^{[1]}$.
However, the theory of dipole source is only applicable when the transceiver distance is far larger than the size of source. When the transceiver distance is comparable to or even smaller than the source size, the dipole theory is not applicable any more, otherwise, the generated result would have limited resolution, although it is consistent with the intensity of farfield radiation. The major limitation is that, integration cannot provide solutions to accurately estimate the near-field radiation as well as the high-order terms ignored in the equation of computing far-field radiation ${ }^{[2-3]}$.

In most cases, the result accuracy is acceptable by computing the field response through the integration of a series of dipole sources. However, with the increasing requirements on the accuracy of TEM surveying, it is expected to introduce the concept of infinitesimal point charge and develop a new method for response computation, so that the subtle errors due to dipole micro-unit could be effectively eliminated.

Monopole antenna is about half of the dipole antenna. By setting a monopole antenna on the plane of the conductor, radiation is induced by the monopole antenna on the conductor Based on the imaging theory, it is capable of generating the radiation similar to that of a dipole antenna. One strength is that the fed point locates at the top of the antenna, and thereby the monopole antenna has better directivity than the dipole antenna. In such case, the antenna should not be considered as a dipole that consisted of two equivalent charges with opposite polarity, but a monopole that would generate radiation when the charge is accelerated and starts moving. Martin (1999) compared the transmission between the dipole antenna and the traveling-wave antenna, and his conclusion is that the radiation by dipole antenna at the center is different from that by accelerated-moving charge at the endpoint of the travelingwave antenna ${ }^{[4]}$. Traditionally, the relative motion between two equivalent charges with opposite polarity is regarded as the fundamental of the dipole theory of antenna, and the radiation of a dipole source can be estimated by stacking the fields from all micro charges induced by impulse. Since the dipole source consists of a set of point charges, point charge is more fundamental and mathematic as well as physical relationship can be built through the research on current with point charges and the accelerated-moving point charge.

This paper first proposes a new coordinate system in motion, in which the charge is stationary and the velocity excited by charge at uniform motion can be easily estimated. Then the Lenard - Wishel potential is applied to compute the 
field from charge at accelerated motion, and further the field from electric dipole. Finally, the relationship is investigated between the acceleration field and velocity field from the charge at accelerated motion and the induced field from electric dipole. This paper also develops the equation for computing the transient field from electric dipole and comparing the differences between fields generated from charges at uniform motion and accelerated motion.

\section{POINT CHARGE INTERPRETATION OF HARMONIOUS FIELD FROM ELECTRIC DIPOLE}

Oscillating dipole is the simplest system of generating electromagnetic radiation, which is equivalent to an element with alternating current. The actual antenna can be considered as a series of discrete alternating current element (or element antenna); meanwhile, dipole is a pair of equivalent charges with opposite polarities at periodic motion. When the element antenna is electrified by alternating current, the motion of both charges will be accelerated and produce elemental electric current that induces electromagnetic field in the surrounding space. The traditional electromagnetic field induced by dipole could be interpreted as two special cases. If the transceiver distance is far less than the primary wavelength, observed is the near-field electromagnetic radiation, also known as quasistatic field or induction field. In contrast, if the transceiver distance is far greater than the primary wavelength, observed is the far-field electromagnetic radiation, also known as radiation field. The equivalence between charge source and current source is often expressed as follows

$$
\begin{aligned}
& q=\int I d t=\int I_{0} e^{i \omega t} d t=\frac{I}{i \omega} \\
& p=q l=\frac{I l}{i \omega}
\end{aligned}
$$

Generally, there are two ways of interpreting the charge source of dipole. The first one is the variation of charge and its relative reciprocating motion within the element antenna. In such case,

$$
\begin{aligned}
& q=q_{0} \cos (\omega t) \\
& I=\frac{\partial q}{\partial t}=-q_{0} \omega \sin (\omega t) \\
& p=q_{0} l
\end{aligned}
$$

The second interpretation is the L-type oscillation with constant charge, which is closely related to the acceleration of charge:

$$
q a(t)=q\left(\frac{l^{\prime \prime}}{2}\right)+(-q)\left(-\frac{l^{\prime \prime}}{2}\right)=q l^{\prime \prime}=p^{\prime \prime}=\left(P_{0} \cos (\omega t)\right)^{\prime \prime}=-\omega^{2} P_{0} \cos (\omega t)
$$

In order to build reliable connection between the charge at acceleration motion and the field from dipole, this study adopts the second interpretation to investigate the generation of the dipole field.

\section{A. Radiation field}

As the basic model of radiation generation from lowvelocity motion of the charge, the electric dipole radiation is the most fundamental electromagnetic radiation and can be treated as harmonic oscillation of the dipole. As harmonic oscillation is a special case of linear motion with varying velocity, the electric dipole radiation can then be considered as a special case of charge radiation at acceleration motion.

The radiation field from dipole can be computed as,

$$
E_{\theta}=\frac{I_{0} l K^{2}}{4 \pi \varepsilon_{0} \omega r} e^{i[\omega(t-r / c)+\pi / 2]} \sin \theta
$$

Substituting $K=\frac{2 \pi}{\lambda}=\frac{\omega}{c} ; i=e^{i \pi / 2}$ and Equation (1) into Equation (4) leads to

$$
E_{\theta}=\frac{P_{0} \omega^{2}}{4 \pi \varepsilon_{0} c^{2} r} e^{i[\omega(t-r / c)]} \sin \theta
$$

Compared to the relative motion of charge in the dipole, the charge is at low-velocity motion, and its velocity $V<<c$ The fundamental of dipole lies in the principle $l<<=\mid \begin{array}{rr}\mathrm{V} & \mathrm{v} \\ \mathrm{V} & R^{\prime}\left(t^{\prime}\right) \mid\end{array}$

$$
\left|\mathrm{V}-R_{0}\left(t^{\prime}\right)\right| \approx\left|\mathrm{V}-\stackrel{\mathrm{V}}{R^{\prime}}\left(t^{\prime}\right)\right|=r \quad, \quad \theta^{\prime}=\theta .
$$

Then we can obtain,

$$
E_{\theta}=q a(t-r / c) \sin \theta / 4 \pi \varepsilon_{0} c^{2} r
$$

Which can be simplified by substituting it with equation (3), and the simplified equation is

$$
E_{\theta}=\omega^{2} P_{0} \cos \omega(t-r / c) \sin \theta / 4 \pi \varepsilon_{0} c^{2} r
$$

Note that the real part of Equation (5) is the same as Equation (6).

\section{B. Induction field}

The oscillation of dipole induces not only the radiation field, but also the induction field, which can be computed as,

$$
E_{\theta}=\frac{p \sin \theta}{4 \pi \varepsilon_{0} r^{3}}
$$

In the induction field, the fundamental of dipole remains valid, $l<<r$ and $v<<c$. Both are used for simplifying the velocity field generated from charge at uniform motion.

$$
\stackrel{\mathrm{v}}{E} \approx \frac{q}{4 \pi \varepsilon_{0} r^{3}} \stackrel{\mathrm{v}}{r}
$$


The electric field induced by dipole can be approximated as the field induced by a pair of equivalent charges with opposite polarities at relative motion

$$
\stackrel{\mathrm{v}}{E}=\frac{q_{+}}{4 \pi \varepsilon_{0} r_{+}^{3}} \stackrel{\mathrm{v}}{r_{+}}+\frac{q_{-}}{4 \pi \varepsilon_{0} r_{-}^{3}} \stackrel{\mathrm{v}}{r}
$$

Let $l<<r$, then

$$
r_{+} \approx r-\frac{1}{2} l \cos \theta, r_{-} \approx r+\frac{1}{2} l \cos \theta
$$

and :

$$
\begin{gathered}
r_{-}-r_{+} \approx l \cos \theta, r_{-}+r_{+} \approx 2 r, r_{-} r_{+} \approx r^{2} \\
r_{-}^{3}-r_{+}^{3} \approx 3 r^{3} l \cos \theta, r_{-}^{3}+r_{+}^{3} \approx 2 r^{3}
\end{gathered}
$$

which are then used for simplifying Equation (9). The simplified equation is

$$
E_{\theta}=\frac{p \sin \theta}{4 \pi \varepsilon_{0} r^{3}}
$$

Apparently there is close correlation between the velocity field from moving charge and the induction field from dipole. Therefore, it is more fundamental for interpreting the dipole field through the motion of charges.

\section{MOVING CHARGE INTERPRETATION OF TRANSIENT FIELD FROM ELECTRIC DIPLOE}

It has built the correlation between the field from oscillating dipole and the field from charge at acceleration motion as well as uniform motion. As mentioned above, dipole is a pair of equivalent charges with opposite polarities at periodic motion. When the element antenna is electrified by alternating current, its charge will be accelerated and produce elemental electric current that induces electromagnetic field in surrounding space. Therefore, if the element antenna is electrified with step current, there should also be some correlation between the field from transient dipole and the field from moving charge.

In classic electromagnetic theory, the transient field can be obtained by performing frequency-time transform on the harmonious field, and this section provides the derivation of transient electric dipole field in details.

\section{A. Radiation field}

Equation (4) is often used for computing the radiation field from the harmonious electric dipole.

$$
E_{\theta}=\frac{I_{0} l K^{2}}{4 \pi \varepsilon_{0} \omega r} e^{i[\omega(t-r / c)+\pi / 2]} \sin \theta
$$

Based on $K^{2}=\mu_{0} \varepsilon_{0} \omega^{2} ; i=e^{i \pi / 2}$, the equation can be further simplified as

$$
E_{\theta}=\frac{I_{0} l \mu_{0} i \omega}{4 \pi r} e^{i \omega(t-r / c)} \sin \theta
$$

Let $b=i \omega$, then it can be expressed as

$$
E_{\theta}=\frac{I_{0} l \mu_{0} b}{4 \pi r} e^{b(t-r / c)} \sin \theta
$$

Therefore, the radiation field of the transient electric dipole is computed as

$$
e_{\theta}=L^{-1}\left\{\frac{E_{\theta}}{b}\right\}=L^{-1}\left\{\frac{I_{0} l \mu_{0}}{4 \pi r} e^{b(t-r / c)} \sin \theta\right\}
$$

Application of Laplace transformation

$$
L^{-1}\left\{e^{-\alpha b}\right\}=\delta(t-\alpha) \text { leads to }
$$

$$
e_{\theta}=\frac{I_{0} l \mu_{0}}{4 \pi r} \sin \theta \delta\left(t+t_{0}-r / c\right)
$$

Different from the harmonious field, transient field has the causality on time. $t_{0}$ denotes the time that the charge move to the endpoint of the dipole and $t_{0}=\frac{L}{v} . t$ denotes the observation time.

Generally, the dipole induces at one endpoint and radiates at the other point. Considering the case that the element antenna radiates at its center, Equation (11) can be further expressed as

$$
e_{\theta}=\frac{I_{0} l \mu_{0} \sin \theta}{4 \pi r}\left[\delta\left(t+\frac{L}{2 v}-\frac{2 r-L \cos \theta}{2 c}\right)-\delta\left(t-\frac{L}{2 v}-\frac{2 r+L \cos \theta}{2 c}\right)\right]
$$

which indicates that the radiation field of the transient dipole consists of two impulses with opposite signs, with one induced at time $t=-\frac{L}{2 v}$ and arriving at point $\mathrm{P}$ after a delay of $\frac{2 r+L \cos \theta}{2 c}$, and the other induced at time $t=\frac{L}{2 v}$ and arriving at point $\mathrm{P}$ after a delay of $\frac{2 r+L \cos \theta}{2 c}$.

If interpreting from the aspect of charge at motion with $+\mathrm{q}$ moving towards $-\mathrm{q}$ with velocity $V$ at time $t=-\frac{L}{2 v}$ and stopped when reaching $-\mathrm{q}$ at time $t=\frac{L}{2 v}$, a current element is induces and then disappears during the period $\left(-\frac{L}{2 v}, \frac{L}{2 v}\right)^{[5]}$. The first impulse is induced when the charge starts it motion, while the second impulse is induced when the motion of the charge stops, both of which indicate that the radiation field is induced by the acceleration motion of the charges

\section{B. Induction field}

Equation (4) is often used for computing the induction field from harmonious electric dipole: 


$$
E_{\theta}=\frac{p \sin \theta}{4 \pi \varepsilon_{0} r^{3}}
$$

Based on $p=I l=I_{0} l e^{i \omega t}$, it can be expressed as

$$
E_{\theta}=\frac{I_{0} l e^{i \omega t} \sin \theta}{4 \pi \varepsilon_{0} r^{3}}
$$

Let $b=i \omega$, then

$$
E_{\theta}=\frac{I_{0} l e^{b t} \sin \theta}{4 \pi \varepsilon_{0} r^{3}}
$$

Therefore, the induction field of the transient electric dipole is computed as

$$
e_{\theta}=L^{-1}\left\{\frac{E_{\theta}}{b}\right\}=L^{-1}\left\{\frac{I_{0} l e^{b t} \sin \theta}{4 \pi \varepsilon_{0} r^{3} b}\right\}
$$

Application of Laplace transform $L^{-1}\left\{\frac{e^{-\alpha b}}{b}\right\}=u(t-\alpha)$ leads to

$$
e_{\theta}=\frac{I_{0} l \sin \theta}{4 \pi \varepsilon_{0} r^{3}} u\left(t+\frac{l}{v}\right)
$$

Considering the case that the element antenna induces at its center. Equation (12) can be further expressed as

$$
e_{\theta}=\frac{I_{0} l \sin \theta}{4 \pi \varepsilon_{0} r^{3}}\left[u\left(t+\frac{l}{2 v}\right)-u\left(t-\frac{l}{2 v}\right)\right]
$$

Numeric consistency is noticed between the self-field from transient electric dipole and the field from constant current. However, the inducing time of the source is limited in the period $\left(-\frac{L}{2 v}, \frac{L}{2 v}\right)$, which is the same as the period when the charge is at uniform-motion.

\section{CONCLUSION}

The dipole solution is a special case of estimating the farfield radiation in antenna theory; however when applied to near-source TEM observation, it could cause significant numeric errors. Therefore, the charge at acceleration motion provides a better interpretation of the physical mechanism of the induction field generated from actual antenna, which is not only more accurate in computation but also more reasonable in theory.

\section{REFERENCES}

[1] Nabighian, M. N., 1988. Electromagnetic methods in applied geophysics, Volume 1: Society of Exploration Geophysicists, Tusla, 203-252.

[2] Xue, G. Q., Yan, S., and Zhou, N. N., 2011. Theoretical study on the error caused by dipole hypothesis of large loop TEM response: Chinese Journal of Geophysics (in Chinese), 54, 2389-2396.

[3] Yan, S., Xue, G. Q., and Chen, M. S., 2011. Review and perspective of theoretical study on large-loop TEM response: Progress in Geophysics (in Chinese), 26, 941-947.

[4] Martin, R. G., 1999. Some thoughts about transient radiation by straight thin wires: IEEE Antennas and Propagation Magazine, 41, 24-33.

[5] Wang, C. Q., and Zhu, X. L., 2011.Theory and calculation of transient electromagnetic field. Beijing: Press of Beijng University, 42-53. 